\title{
Importância da prática de atividade física para as pessoas com autismo
}

\section{Importance of physical activity practice for people with autism}

\author{
Renata Pereira de Aguiar ${ }^{1}$, Fabiane Silva Pereira ${ }^{2}$, Claudiana Donato Bauman ${ }^{3,4}$
}

1. Discente do curso de Educação Física pela Universidade Estadual de Montes Claros (Unimontes), Montes Claros, MG, Brasil. 2. Discente do curso de Medicina pela Universidade Estadual de Montes Claros (Unimontes), Montes Claros, MG, Brasil. 3. Docente da Universidade Estadual de Montes Claros (Unimontes), Montes Claros, MG, Brasil. 4. Doutoranda pelo Programa de Pós-graduação em Ciências da Saúde pela Estadual de Montes Claros (Unimontes), Montes Claros, MG, Brasil.

\section{Resumo}

Introdução: 0 termo "autismo" foi utilizado para caracterizar a perda de contato com a realidade, gerando dificuldade ou impossibilidade de comunicação. Hoje, o autismo faz parte dos transtornos globais do desenvolvimento, chamados de Transtornos do Espectro Autista (TEA). Os autistas necessitam de cuidados multidisciplinares; o tratamento envolve técnicas de mudança de comportamento, programas educacionais ou de trabalho, além de terapias de linguagem/comunicação. Objetivo: 0 objetivo deste estudo foi realizar uma revisão literária de trabalhos que apontassem a importância da prática de atividades físicas para o desenvolvimento de pessoas com TEA, apresentando exemplos de atividades já executadas com essa finalidade, e quais os resultados obtidos. Métodos: 0 presente estudo trata-se de uma revisão de literatura narrativa sobre a importância da prática de atividades físicas por parte de pessoas com autismo, e como esta variável pode auxiliar seu desenvolvimento. Resultados: Estudos demonstram que, por meio da prática de exercícios como caminhada, equinoterapia, e atividades aquáticas as pessoas com autismo conseguem desenvolver melhor sua capacidade comunicativa, reduzir o comportamento antissocial, diminuir comportamentos que demonstram inadaptação, estereotipias e agressividade. Conclusão: Apesar de ser um tema extremamente importante, não se encontram muitos estudos que abordam essa temática; por esse motivo, o presente trabalho adquire relevância e abre espaço para que outras pesquisas sobre o assunto sejam realizadas, levando a um maior conhecimento sobre o tema. Dessa forma, as pessoas com autismo poderão ser mais bem atendidas em todas as suas necessidades biopsicossociais.

Palavras-chave: Benefícios. Autismo. Atividade física.

\begin{abstract}
Introduction: The term "autism" was used to characterize the loss of contact with reality, generating difficulty or impossibility of communication. Today, autism is part of the global developmental disorders, called Autism Spectrum Disorders (ASD). Autistics need multidisciplinary care; Treatment involves behavior change techniques, educational or work programs, as well as language / communication therapies. Objective: The objective of this study was to carry out a literary review of papers that pointed out the importance of the practice of physical activities for the development of people with ASD, presenting examples of activities already performed for this purpose, and the results obtained. Methods: The present study is a review of narrative literature about the importance of physical activity practice by people with autism, and how this variable can help their development. Results: Studies show that, through the practice of exercises such as walking, equine, and aquatic activities, people with autism can better develop their communicative capacity, reduce antisocial behavior, reduce behaviors that show maladaptation, stereotypy and aggressiveness. Conclusion: Although it is an extremely important topic, there are not many studies that address this theme; For this reason, the present work acquires relevance and opens space for other researches on the subject to be carried out, leading to a greater knowledge on the subject. In this way, people with autism can be better served in all their biopsychosocial needs.
\end{abstract}

Key words: Benefits. Autism. Physical activity.

\section{INTRODUÇÃO}

O termo "autismo" foi citado pela primeira vez por Bleuler em 1911, para caracterizar a perda de contato com a realidade, gerando dificuldade ou impossibilidade de comunicação ${ }^{1}$.

Em 1943, o psiquiatra austríaco Leo Kanner usou esse termo para designar uma doença caracterizada por isolamento extremo, alterações de linguagem marcadas pela ausência de finalidade comunicativa, rituais do tipo obsessivo com tendências à mesmice, movimentos estereotipados e déficit na coordenação motora² .

Hoje, o autismo faz parte dos transtornos globais do desenvolvimento, chamados de Transtornos do Espectro Autista (TEA) que, de acordo com o último Manual de Saúde Mental
(DSM V) inclui o autismo, transtorno desintegrativo da infância e as síndromes de Asperger e Rett ${ }^{3}$. O TEA pode ser identificado desde os primeiros anos de vida, e se caracteriza por distúrbios na tríade: interação social, comunicação, e reciprocidade social (com interesses peculiares e padrões estereotipados do comportamento $)^{4}$.

Os portadores do TEA podem apresentar diferentes características; alguns têm dificuldade de aprendizagem em vários sentidos, até mesmo em relação às atividades de vida diária, como tomar banho e fazer a própria refeição, enquanto outros poderão levar uma vida relativamente "normal", com poucas limitações. Mas é preciso ter em mente que o autismo é uma condição permanente que carece atenção ${ }^{1}$.

Correspondência: Fabiane Silva Pereira. Universidade Estadual de Montes Claros (Unimontes), Montes Claros, Minas Gerais, Brasil. E-mail: fabiane. silvapereira1@gmail.com

Conflito de interesse: Não há conflito de interesse por parte de qualquer um dos autores.

Recebido em: 26 Dez 2016; Revisado em: 24 Jan 2017; 9 Mar 2017; Aceito em: 13 Mar 2017 
Os autistas necessitam de cuidados multidisciplinares; o tratamento envolve técnicas de mudança de comportamento, programas educacionais ou de trabalho, além de terapias de linguagem/comunicação ${ }^{1}$. O objetivo dessas abordagens é melhorar a aquisição da linguagem, as habilidades de interação social e diminuir os comportamentos mal-adaptativos ${ }^{5}$.

Não existem tratamentos medicamentosos aprovados; porém, podem ser utilizadas drogas para sintomas específicos, como agressividade e hiperatividade ${ }^{5}$.

Um sinal marcante dos indivíduos autistas é sua tendência ao isolamento. Em geral, quanto maior o comprometimento cognitivo, maior a chance de se isolar e não se comunicar, uma vez que esses indivíduos têm dificuldade em compreender as interações sociais ${ }^{6}$.

Esse isolamento pode trazer ainda mais prejuízos para a vida dessas pessoas, atrapalhando sua interação social e dificultando a vida profissional. Tentando minimizar tais danos, alguns pesquisadores têm demonstrado a importância da atividade física para as pessoas com TEA.

Por meio da atividade física, os pacientes podem vencer a ociosidade e a baixa capacidade de iniciativa, desenvolvendo, assim, uma interação social mais adequada, melhora da coordenação motora e da capacidade cognitivo-emocional, além de desenvolver a consciência corporal e espaço-temporal².

Este estudo adquire relevância por apresentar uma nova forma de auxiliar cuidadores, profissionais e familiares no manejo de indivíduos autistas, pois mesmo que os medicamentos sejam, às vezes, necessários, outras formas de abordagem também podem ser utilizadas, desde que se mostrem benéficas.

Nesse sentido, o presente estudo teve como objetivo fazer uma revisão literária de estudos que apontem a importância da prática de atividades físicas para o desenvolvimento de pessoas com TEA, apresentando estudos que mostrem quais atividades já foram executadas com essa finalidade, se existe algum tipo de exercício que seja mais adequado que outro, em se tratando de indivíduos autistas, e quais foram os resultados encontrados em relação ao desenvolvimento psicossocial dessas pessoas.

\section{MÉTODOS}

O presente estudo tem por finalidade fazer uma revisão de literatura narrativa; para tal, ele faz uma seleção crítica de publicações sobre um assunto específico que, nesse caso, trata da importância da prática de atividades físicas por parte de indivíduos com autismo e, como esse fato pode auxiliar em seu desenvolvimento?.

\section{Critérios de inclusão}

Para compor a amostra da presente pesquisa foram utilizados os seguintes critérios de inclusão: artigos publicados nos últimos 16 anos (2001 a 2016); disponíveis na íntegra nas bases de dados SciELO (Scientific Eletronic Library Online) e PubMed, publicados nas línguas português, inglês ou espanhol, e que tratassem do tema proposto. Foram excluídos os artigos que não obedeciam a esses critérios.

\section{Procedimentos de pesquisa}

Foi feita uma busca de artigos nas bases de dados SciELO e PubMed, utilizando os descritores: autismo, benefícios, atividade física, bem como combinações entre eles. Optouse por realizar buscas com o termo autismo isoladamente e em combinação com os outros dois (benefícios e atividade física), uma vez que a busca isolada com os outros descritores mostrava trabalhos que não contemplavam o assunto principal desta pesquisa, que é o transtorno do espectro autista.

$\mathrm{Na}$ base de dados SciElO foram encontrados para o termo "autismo" 435 publicações; para a busca combinada "autismo + atividade física" três publicações; "autismo + benefícios" três publicações, totalizando 441 estudos. Foi feita uma leitura dos títulos de todos esses trabalhos, selecionando-se 35 artigos. Realizou-se, então, a leitura dos resumos para avaliar se os trabalhos eram condizentes com o tema desta pesquisa e, com base nisso, foram selecionadas 10 publicações (Figura 1).

Figiura 1: Busca de acordo com os descritores e critérios utilizados para a escolha de artigos. Na base de dados PubMed foram desconsiderados os materiais encontrados com 0 descritor "Autismo" devido ao grande volume de publicações encontradas.



J. Health Biol Sci. 2017; 5(2): 178-183 
Já na base de dados PubMed foram encontradas: para o termo "autismo" 11448 publicações; "autismo + atividade física" 109 publicações; "autismo + benefícios" 158 publicações. Devido ao grande número de publicações encontradas para o descritor "autismo" e o tempo necessário para a realização do presente trabalho, optou-se por utilizar os resultados das buscas "autismo + atividade física" e "autismo + benefícios", totalizando 267 publicações. Realizou-se então a leitura dos títulos desses trabalhos, selecionando-se 25 publicações que tiveram seus resumos lidos, e a partir disso, foram escolhidas seis publicações (Figura 1).

Por fim, foram selecionados 16 trabalhos que foram lidos na íntegra e utilizados para a elaboração do presente estudo.

Vale frisar que, além das 16 publicações selecionadas, também foi utilizado o livro "Como escrever um trabalho científico" disponível no portal da Sociedade Brasileira de Ortopedia e Traumatologia, e um artigo sobre o DSM-V (disponível na base de dados SciELO). Totalizando assim 18 publicações utilizadas.

\section{Aspectos éticos}

O projeto foi aprovado pelo Comitê de Ética em Pesquisa da Unimontes, parecer consubstanciado no 1866468 de 2016.

\section{RESULTADOS E DISCUSSÃO}

O autismo consiste em um distúrbio neurológico caracterizado por baixa interação social, limitações na comunicação, além de movimentos repetitivos; geralmente essas alterações se manifestam nos primeiros anos de vida ${ }^{8}$.

O autismo tem uma prevalência estimada em 10 a 13 pessoas por 10.000 indivíduos. Existem várias possíveis causas para esse transtorno; sendo a herança genética amplamente defendida, uma vez que a concordância entre gêmeos monozigóticos pode chegar a $60 \%$; além disso, a história familiar positiva também aumenta a chance de surgimento da doença. Mas é preciso salientar que a alteração genética por si só não leva ao desenvolvimento do autismo, é necessário que haja interação com outros fatores ambientais, que ainda não estão bem definidos pela literatura?.

Já foram realizados estudos pós-morte em indivíduos sabidamente autistas, os quais evidenciaram alterações em regiões cerebrais, como volume reduzido do giro cingulado posterior e anterior direito, menor número de células de Purkinje no cerebelo, e também menor número de células nas estruturas do sistema límbico ${ }^{10}$.

Exames de imagens também são úteis para identificar alterações cerebrais presentes nas pessoas com TEA; entretanto, ainda não existe um marcador genético ou uma alteração específica que confirme a doença. Por isso, o diagnóstico do autismo é baseado em critérios comportamentais ${ }^{10}$.
As alterações clínicas compatíveis com o autismo (como prejuízo na comunicação, interação social e uso da imaginação) podem ser percebidas antes dos três anos de idade; e quanto mais precoce o diagnóstico, melhores são os resultados das intervenções realizadas ${ }^{11}$.

Não existe nenhum tratamento totalmente eficaz, mas o foco das medidas terapêuticas é melhorar a habilidade de interação social e de linguagem, além de atenuar os comportamentos caracterizados por inadaptação. Por isso, é necessária uma abordagem multidisciplinar, com fonoaudiólogos, psicólogos, educadores físicos e médicos. Também pode ser necessário um tratamento medicamentoso, a depender do quadro clínico do paciente ${ }^{11}$.

Como mencionado, não existe um único tratamento para o quadro, mas sim diversas formas de abordá-lo. Pensando nisso, alguns estudiosos têm demonstrado a importância da atividade física para o bom desenvolvimento das habilidades dos autistas. Nesse sentido, foi realizado um estudo ${ }^{12}$ com 16 meninos autistas, entre 6 e 9 anos de idade, que foram estimulados a realizar cerca de 90 minutos semanais de atividades aquáticas durante 10 semanas. O estudo foi realizado da seguinte forma: durante 10 semanas consecutivas um grupo de 8 (oito) meninos realizou o plano atividades aquáticas, ao final foram avaliados quanto à aquisição de habilidades de natação e interação social. Em seguida, o outro grupo de 8 (oito) meninos foi submetido ao mesmo plano de exercícios e avaliados ao final. Após 10 semanas, todos foram reavaliados, e observou que houve melhora das habilidades aquáticas, e redução do comportamento antissocial. Entretanto, não houve nenhuma progressão após a cessação do programa, ou seja, a realização contínua de atividades físicas contribui significativamente para o melhor desenvolvimento das pessoas com TEA.

A prática de exercícios físicos consegue atenuar várias características das pessoas com autismo, como padrões de comportamento de inadaptação, comportamentos estereotipados e agressivos ${ }^{8}$.

Em um estudo ${ }^{13}$ envolvendo 34 indivíduos com TEA, foi criado um grupo experimental, formado por 2 (duas) meninas e 17 meninos - entre 5 a 10 anos de idade; e um grupo controle, composto por 3 (três) meninas e 12 meninos - entre 4 a 10 anos de idade. $O$ grupo experimental foi submetido à prática de equitação terapêutica, uma hora por semana, durante 3 (três) meses. Ao final do programa percebeu-se que os participantes que realizaram a atividade apresentaram maior sensibilidade sensorial, motivação social e menos desatenção, distração e comportamentos sedentários, quando comparados ao grupo controle.

Essa melhora comportamental também foi evidenciada por meio de uma pesquisa ${ }^{8}$ em que se analisaram 18 trabalhos que tratavam de programas de abordagem motora (como exercícios aquáticos, corrida, caminhada, jogos e atividades de lazer) envolvendo pessoas autistas entre 2 (dois) e 39 anos de idade; 
foi observado que em todos esses trabalhos houve melhora significativa no comportamento dos indivíduos autistas. Observou-se redução de comportamentos estereotipados, mal adaptativos e antissociais; redução da desatenção; melhora da flexibilidade, equilíbrio e força muscular.

Além das atividades físicas mais comuns, outros tipos de atividade também podem favorecer o desenvolvimento psicossocial dos indivíduos autistas. Isso pode ser evidenciado por meio de uma pesquisa ${ }^{4}$ realizada com 2 (dois) meninos autistas, ambos com 6 anos de idade que cursavam o jardim primário em escolas privadas de ensino regular da cidade de Belém do Pará. Os meninos participaram de aulas de percussão em uma escola de música, cada um deles foi incluído em um grupo diferente. Cada grupo era constituído por 10 crianças [sendo que 5 (cinco) delas se enquadravam dentro do TEA], as aulas ocorreram duas vezes por semana, com duração de 30 minutos, por três meses. Durante as aulas, os alunos escutavam música e aprendiam técnicas de percussão com um professor, que envolviam desde o uso de instrumentos até a movimentação corporal. Ao final do período do estudo, observou-se que as duas crianças apresentaram melhora na interação social, com tendência a tomar iniciativas e à diminuição de comportamentos não funcionais, mostrando, assim, que qualquer atividade que, preferencialmente, seja realizada em grupo, favorece a interação dos indivíduos autistas.

As artes marciais também podem ser úteis no processo de desenvolvimento dos indivíduos com autismo. Isso pode ser evidenciado por meio de um estudo ${ }^{14}$ realizado com 30 crianças em idade escolar com TEA, em que 15 delas haviam participado de aulas de Karatê por 14 semanas, e as outras 15 representavam o grupo controle e não realizaram as aulas. Todas as crianças foram avaliadas quanto ao déficit de comunicação antes, ao final das 14 semanas e um mês após o término das aulas, e evidenciou-se que as crianças que realizaram as aulas apresentaram melhora na comunicação social em relação ao grupo controle, e esse ganho permaneceu inalterado um mês depois do encerramento das atividades. Daí, percebe-se que os ganhos com os exercícios não são apenas temporários, mas acompanham o indivíduo por toda a sua vida.

Entretanto, apesar de estar bem documentado na literatura, os benefícios da atividade física para os indivíduos com autismo, uma equipe de pesquisadores ${ }^{15}$ chama a atenção para a importância de que esses exercícios sejam programados pensando-se nesse grupo populacional. Esses pesquisadores aplicaram um questionário em 111 pais (58 pais de crianças com desenvolvimento típico, e 53 pais de crianças com TEA), que abordava as barreiras familiares, sociais e comunitárias para a participação de seus filhos em atividades físicas. As crianças tinham entre 3 (três) e 11 anos de idade. Observou-se que $60 \%$ dos pais de indivíduos com autismo relatam que as crianças necessitavam de supervisão excessiva para a realização das atividades, já os pais das crianças em desenvolvimento típico não apresentaram nenhuma queixa neste sentido. Além disso, os pais de indivíduos com TEA relataram a exclusão das crianças por outras (com desenvolvimento típico) e a dificuldade dos adultos em incluir os autistas nas atividades, o que evidencia a importância de conhecer as limitações de cada criança antes de propor um plano de atividades, e também a absoluta necessidade de se educar as outras crianças para evitar discriminações.

Como já foi dito, os indivíduos com autismo tendem a ficar mais isolados e interagirem pouco com as pessoas ao redor, isso aumenta muito o risco de sobrepeso. Estima-se ${ }^{16}$ que a prevalência de obesidade em crianças com TEA é de 30,4\%; já nas crianças de mesma idade e sem TEA é de 23,6\%. Outros fatores que contribuem para isso são a má nutrição, deficiências motoras e de coordenação, deficiências cognitivas, uso de medicamentos (como antipsicóticos e antidepressivos), anormalidades metabólicas, e falta de conhecimento a respeito desse problema ${ }^{16}$. Por isso, antes de elaborar um plano de atividade física deve-se ter como meta melhorar as deficiências específicas do autismo, bem como auxiliar na redução do sobrepeso. Para tanto, podem ser combinados exercícios de resistência, flexibilidade, aeróbica, com atividades em grupo ou aos pares, sempre levando em consideração as necessidades individuais ${ }^{16}$.

Visando observar o efeito da atividade física sobre o desempenho escolar, foi feita uma pesquisa ${ }^{17}$ com quatro crianças de nove anos de idade, que praticaram 12 minutos de corrida, três vezes por semana, durante duas semanas. Ao final da pesquisa percebeu-se que as crianças tinham melhorado significativamente seu desempenho nas atividades escolares, mostrando assim a importância da prática de atividades físicas para os indivíduos com TEA.

As crianças autistas podem e devem participar das aulas de educação física ministradas na escola; entretanto, é preciso ter atenção a determinadas peculiaridades desse grupo de indivíduos. $\mathrm{O}$ autista pode apresentar crises de agressividade quando percebe que sua rotina foi quebrada; por isso, as aulas devem ser ministradas em horário fixo, com duração determinada e os objetos utilizados devem ser distribuídos de maneira lenta, para que a criança se adapte à situação ${ }^{18}$.

Conforme demonstrado em estudos, apesar de as pessoas com autismo terem necessidades especiais quanto a seu aprendizado e relações interpessoais, elas são iguais a todas as outras; como tal, precisam de atenção e estímulo para desenvolver suas habilidades, principalmente em relação à comunicação. Nesse aspecto, a atividade física é de grande valia, pois, durante essas atividades, os autistas são estimulados a se expressar e conviver em grupo, melhorando, assim, sua capacidade de interação e comunicação.

\section{CONCLUSÃO}

As pessoas com autismo possuem características que precisam ser conhecidas e respeitadas. A partir daí, os profissionais conseguem estabelecer planos de atividades físicas que esses 
indivíduos possam realizar, e por meio deles, desenvolver suas habilidades e atenuar características como o comportamento agressivo e estereotipado.

Foram encontrados poucos trabalhos relacionando a realização de atividade física por pessoas com autismo aos benefícios advindos dessa prática, - mas todos os analisados salientam os efeitos positivos dessas atividades.

Contudo, apesar de os resultados serem muito positivos, é preciso fazer algumas observações. Entre os estudos analisados, observa-se que a maioria envolve principalmente crianças, predominantemente do sexo masculino, o que pode gerar algum viés, pois as meninas podem apresentar preferências diferentes daquelas demonstradas pelos meninos, o que deve ser levado em consideração; já os adultos podem ter uma resposta diferente aos exercícios em relação às crianças, como maior dificuldade de adaptação e mais tempo para apresentar mudanças no comportamento e em outras áreas de sua vida.

Outra observação a ser feita é quanto ao número de participantes, que não é muito grande; isso torna a execução das atividades mais fácil, entretanto interfere na generalização dos resultados. Também é preciso destacar a ausência de grupo controle na maioria dos estudos, que poderia servir para dar maior embasamento aos resultados.

Além disso, observa-se que a quase totalidade das pesquisas visava à melhora dos padrões de comportamento, alterações motoras, interação social e atenção dos indivíduos autistas; em apenas um dos trabalhos foi levantada a questão da obesidade, muito frequente nesse grupo populacional devido ao maior isolamento e ociosidade.

Outro assunto a ser frisado, é que a maioria dos estudos apresenta atividades realizadas em grupo, mas, devido à maior tendência ao isolamento, pode ser que inicialmente seja necessário que elas aconteçam individualmente, e com o tempo e a resposta da pessoa com TEA, seriam introduzidos os exercícios em grupo.

É possível inferir que, apesar dos vários efeitos benéficos dos exercícios físicos para a vida das pessoas com autismo, não existe um plano de exercícios que seja ideal, mas sim que ele deve ser estabelecido com base nas características individuais e nos objetivos que se pretende alcançar.

Por isso, é importante conhecer as limitações físicas, psíquicas e sociais das pessoas com TEA, o que pode ser feito por meio da aplicação de questionários, realização de testes como atividades em grupo e exames para avaliar a capacidade motora; também podem ser feitas entrevistas com os indivíduos autistas e seus pais ou familiares. Tudo isso ajuda a traçar o perfil da pessoa, e assim determinar se é preciso realizar planos de atividades que foquem em exercícios de interação social (como natação e aulas de música), exercícios aeróbicos (como corridas), exercícios para melhorar o equilíbrio, diminuir comportamentos estereotipados, diminuir a desatenção; enfim, as atividades físicas a serem propostas devem ser sempre orientadas de acordo com a finalidade almejada.

Vale ressaltar, também, que é primordial avaliar os resultados pós-intervenção comparando com os dados pré-intervenção, analisar a eficácia das atividades, e se é ou não necessário realizar alguma modificação.

Além disso, é preciso frisar que faz parte do quadro clínico do autismo a tendência ao sedentarismo; portanto, estimular a prática de exercícios promove maior interação social, além de garantir melhor qualidade de vida.

Apesar de ser um tema extremamente importante, a dificuldade de encontrar estudos sobre esse assunto, principalmente no Brasil, reflete a importância do presente trabalho e a necessidade de realizar pesquisas com grupos maiores de indivíduos, envolvendo igualmente ambos os sexos, várias faixas etárias e grupos-controle para que, assim, os resultados tenham maior credibilidade.

Além disso, com maior embasamento teórico-prático, será possível estabelecer planos de atividade física pensando nas principais deficiências do autismo (interação social, comunicação e comportamentos de inadaptação). Desta forma, os indivíduos com TEA poderão ser mais bem atendidos em todas as suas necessidades biopsicossociais.

\section{REFERÊNCIAS}

1. Gadia CA, Tuchman R, Rotta NT. Autismo e doenças invasivas de desenvolvimento. J. Pediatr. 2004 Abr; 80(Supl 2): 83-94. doi: http://dx.doi. org/10.1590/S0021-75572004000300011.

2. Santos EO, Faustino PF, Zengo LM, Macedo VP, Seabra MO Jr. Análise das mediações ocorridas a partir da aplicação de um programa de atividades psicomotoras de caráter lúdico-recreativo em crianças autistas. Colloquium Humanarum. 2012 Jul-Dez; 9(Especial): 641-651.

3. Araújo AC, Lotufo F Neto. A Nova Classificação Americana Para os Transtornos Mentais - o DSM-5. Rev. bras. ter. comport. cogn. 2014 Abr; 16(1): 67-82.

4. Nascimento PS, Zanon RB, Bosa CA, Nobre JPS, Freitas AD Jr, Silva SSC. Comportamentos de crianças do espectro do autismo com seus pares no contexto de educação musical. Rev. bras. educ. espec. 2015 Jan-Mar; 21(1): 93110. doi: http://dx.doi.org/10.1590/S1413-65382115000100007.

5. Nikolov R, Jonker J, Scahill L. Autismo: tratamentos psicofarmacológicos e áreas de interesse para desenvolvimentos futuros. Rev. Bras. Psiquiatr. 2006 Maio; 28(Supl 1): S39-46. doi: http://dx.doi.org/10.1590/S151644462006000500006 .

6. Sanini, Cláudia; BOSA, Cleonice Alves. Autismo e inclusão na educação infantil: Crenças e autoeficácia da educadora. Estud. Psicol. (Natal). 2015 JulSet; 20(3): 173-183. doi: http://dx.doi.org/10.5935/1678-4669.20150019.

7. Mercadante, MT. Revisão de literatura. In: Cristante, AF; kfuri, M, coordenadores. Como escrever um trabalho científico. São Paulo: SBOT; 2011. 
8. Lourenço CCV, Esteves MDL, Corredeira RMN, Seabra AFT. Avaliação dos efeitos de programas de intervenção de atividade física em indivíduos com transtorno do espectro do autismo. Rev. Bras. Ed. Esp. 2015 Abr- Jun; 21(2): 319-328. doi: http://dx.doi.org/10.1590/S1413-65382115000200011.

9. Gupta AR, State MW. Autismo: genética. Rev Bras Psiquiatr. 2006; 28(Supl 1): S29-38.

10. Garcia PM; Mosquera CFF. Causas neurológicas do autismo. O Mosaico. 2011 Jan-Jun; (5).

11. Mesquita WS, Pegoraro RF. Diagnóstico e tratamento do transtorno autístico em publicações brasileiras: revisão de literatura. J Health Sci Inst. 2013; 31(3): 324-9.

12. Pan CY. Effects of water exercise swimming program on aquatic skills and social behaviors in children with autism spectrum disorders. Autism. 2010 Jan; 14(1): 9-28. doi: 10.1177/1362361309339496. PubMed PMID: 20124502.

13. Bass MM, Duchowny CA, Llabre MM. The effect of therapeutic horseback riding on social functioning in children with autism. J Autism Dev Disord. 2009 Sept; 39(9): 1261-1267. doi: 10.1007/s10803-009-0734-3. PubMed PMID: 19350376.
14. Bahrami F, Movahedi A, Marandi SM, Sorensen C. The effect of karate techniques training on communication deficit of children with autism spectrum disorders. J Autism Dev Disord. Mar 2016; 46(3): 978-986. doi: 10.1007/s10803015-2643-y. PubMed PMID: 26577688.

15. Must A, Phillips S, Curtin C, Bandini LG. Barriers to physical activity in children with autism spectrum disorders: relationship to physical activity and screen time. J Phys Act Health. 2015 Apr; 12(4): 529-534. doi: 10.1123/ jpah.2013-0271. PubMed PMID: 25920014.

16. Srinivasan SM, Pescatello LS, Bhat AN. Current perspectives on physical activity and exercise recommendations for children and adolescents with autism spectrum disorders. Phys Ther. 2014 Jun; 94(6): 875-889. doi: 10.2522/ ptj.20130157. PubMed PMID: 24525861.

17. Nicholson H, Kehle TJ, Bray MA, Heest JV. The effects of antecedent physical activity on the academic engagement of children with autism spectrum disorder. Psychol Sch. 2011; 48(2):198-213.

18. Marqueze L; Ravazzi L. Inclusão de autistas nas aulas de educação física. In: Anais do VII Encontro da associação brasileira de pesquisadores em educação especial; 2011 Nov 8-10; Londrina, 2011. p. 1945-1956.

\section{Como citar este artigo/How to cite this article:}

Aguiar RP, Pereira FS, Bauman CD. Importância da prática de atividade física para as pessoas com autismo. J Health Biol Sci. 2017 Abr-Jun; 5(2): 178-183. 